\title{
LA PAZ TRANSFORMADORA: UNA PROPUESTA PARA LA CONSTRUCCIÓN PARTICIPADA DE PAZ Y LA GESTIÓN DE CONFLICTOS DESDE LA PERSPECTIVA SOCIOPRÁXICA
}

TRANSFORMATIVE PEACE: A PARTICIPATORY PEACE BUILDING AND CONFLICT MANAGEMENT PROPOSAL FROM THE SOCIOPRAXIC PERSPECTIVE

\author{
Manuel Montañés Serrano \\ Esteban A. Ramos Muslera \\ Universidad de Valladolid, Campus de Segovia, España \\ mms@soc.uva.es \\ esteban.ramos@gmail.com \\ Recibido: 05/11/2012 \\ Aceptado: 28/11/2012
}

\begin{abstract}
Resumen
En el artículo se expone un marco epistemológico y teórico-metodológico para la ciencia de la paz y el conflicto. Esta propuesta, desarrollada desde la perspectiva sociopráxica, plantea una conceptualización del conflicto social y la paz como procesos vivos y activos cuyos principales protagonistas son los seres humanos y las redes de relaciones sociales constituidas por y constituyente de las mismos.

De acuerdo con el marco enunciado, los modelos convivenciales violentadores son sustituidos por modelos convivenciales sinérgicos con los que atender las necesidades.

Palabras clave: Paz; Conflicto; Metodologías participativas; Redes, Sociopráxis.

Abstract

The article presents an epistemological, theoretical/methodological framework for peace and conflict. This proposal, developed from the sociopraxic perspective, raises a conceptualization of social conflict and peace as living and active processes whose main protagonists are human beings and the networks of social relations constituted by and constituent of them.
\end{abstract}


In accordance with the proposed framework, convivial models responsible for violence are replaced by synergic convivial models with which to attend needs.

Keywords: Peace, Conflict, Participatory methodologies; Networks, Sociopraxis.

\section{INTRODUCCIÓN}

De igual modo que Cortazar dice que un puente es un hombre cruzando un puente, se podría decir que la paz o el conflicto carece de naturaleza propia sin la presencia de los seres humanos que dan sentido a la realidad vivida. Esta aparente obviedad, parece haber pasado desapercibida a la hora de planificar procesos de construcción de paz y/o gestión de los conflictos, pues, si bien, numerosos autores han realizado un ingente trabajo de intervención práctica en los conflictos, no es menos cierto que lo han hecho sin prestar la atención proritaria que se le debería dar a los principales protagonistas de los mismos, que no son otros que la ciudadanía de base.

La paz y el conflicto son realidades socioculturales construidas, por tanto, se ha de propiciar la participación de los sujetos en la gestión de los conflictos y la construcción de la paz.

En este artículo se aporta un marco epistemológico y teórico que justifican y fundamentan, desde la perspectiva de una propuesta de Paz Transformadora, la necesidad de hacer partícipe a la población en la construcción de propuestas con las que contribuir a la paz y a la gestión de conflictos.

\section{FASES EN LOS ESTUDIOS DE LA PAZ: PAZ NEGATIVA, PAZ POSITIVA Y PAZ TRANSFORMADORA}

El estudio de la paz ha pasado por diferentes fases. En términos de posicionamientos diferenciados, se puede hablar de Paz negativa, positiva, e imperfecta. Esta última es utilizada como antesala referencial de la Paz transformadora.

Primera fase: Paz negativa

En esta primera fase se relaciona directamente el concepto paz con la ausencia de enfrentamientos armados. Tiene su origen en la cultura grecolatina que entiende a la eirene como

un estado de ausencia de guerra o de intermedio entre dos conflictos (...) [siendo además una paz] que se refiere a un estado de tranquilidad aplicada sólo a los grupos griegos y en el interior de éstos: pueblos, ciudades-estado, pero nunca a la colaboración o interrelación entre los griegos y los «bárbaros». La Eirene se relaciona con armonía mental, exterior y anímica que se traduce en sentimientos apacibles (Jiménez, 2009: 147). 
Por su parte, la Pax romana contenía la concepción de ausencia de guerras vinculando la firma de la paz tras la guerra, por lo que se asociaba el concepto paz con el de guerra. El orden y el control, pero no la justicia y la prosperidad (Jiménez, 2009), de lo que se desprende una primera aproximación a esta concepción de paz negativa, que se podría interpretar, de acuerdo con Jiménez remitiéndose a Galtung (1985), como sigue:

la paz como una unidad interior frente a una amenaza exterior (...) [donde] los aparatos militares aparecen como una necesidad de defensa y conquista de la paz y tiene sus expresiones en el desarrollo del militarismo y el armamentismo (nivel nacional), y del imperialismo, expansión colonial y política de pactos y alianzas contra amenazas enemigas (nivel internacional) (Jiménez Bautista, 2009: 147)

y una segunda, vinculada con la concepción eurocéntrica de la paz y el Derecho de Estado (Jiménez, 2009). A lo largo de esta primera fase, se asociará el estudio de la paz, La Peace Research, al de la violencia directa y las confrontaciones bélicas.

\section{Segunda fase: Paz positiva}

Esta segunda fase se enmarca en el concepto de violencia estructural. Fue Galtung, en 1960, quien introdujo la noción de violencia estructural. Según este autor, mientras existan injusticias y no se atiendan las necesidades humanas básicas (bienestar, libertad, identidad y sobrevivencia) (...), no existirá la paz aunque no nos agredamos directamente." (Galtung, 1985).

De acuerdo con este enfoque, el concepto de paz se amplía hacia nuevas consideraciones y campos, tales como la construcción de la justicia social y el desarrollo para que todos los seres humanos puedan atender sus necesidades, los movimientos sociales por los derechos humanos, las reivindicaciones feministas, las manifestaciones en contra de la guerra.

En este marco de estudios para la paz, Galtung u otros autores, como Lederach (1997), proponen un enfoque de intervención en los conflictos, Conflict Transformation, mediante el cual tratar la Peace Building como un proceso vivo y activo, donde no sólo los principales líderes político-militares han de participar en la búsqueda de soluciones negociadas, sino también una representación, más o menos, extensa de la sociedad civil.

Este nuevo paradigma propugna que además de los pertinentes "alto el fuego" o "firmas de la paz" se ha de propiciar un proceso de construcción de paz que hunda sus raíces en los aspectos relacionados con la justicia social y el desarrollo: un conflicto no finaliza con la firma de un tratado de paz sino que requie- 
re de un proceso de intervención en aspectos socioculturales que se han visto asimismo afectados.

El análisis de las cuestiones relacionadas con las causas de los conflictos (lo estructural), los contenidos sociales (las relaciones interpersonales, los sentimientos, los reconocimientos), y también las intervenciones de terceras partes (las mediaciones, arbitrajes) pasan a ser parte del contenido del conflicto y, consecuentemente, de las estrategias para su transformación.

En este sentido, Lederach propone "superar el conflicto mediante la reconciliación de las partes y la reconstrucción de las relaciones humanas" (París y Martínez, 2008: 20). Defiende un modelo basado en los valores de la paz como justicia social frente a las primeras consideraciones de conflict resolution ${ }^{1}$.

Este modelo propone la participación de los principales líderes comunitarios, y su intervinculación, aunque se muestra tibio en cuanto a la incorporación plena de la población de base en estos procesos.

\section{Tercera fase: Paz imperfecta}

En la tercera fase, se pueden considerar múltiples aportaciones, concepciones y propuestas, desde una visión más amplia y compleja, respecto de la Paz y los conflictos. En este amplio marco, lleno de lagunas y arenas movedizas, destacan los modelos derivados del Conflict Transformation de Lederach (1997), la propuesta de paz neutra de Jiménez (2009), o las apuestas desde diferentes instituciones como la Escola de Pau con Vicenç Fisas a la cabeza, el Instituto de paz y conflictos de la Universidad de Granada o el Instituto Interuniversitario de Desarrollo Social y Paz de la Universidad de Alicante, y la de autores como Redorta (2004), o la de Vicent Martínez desde la filosofía de paz, o las aportaciones de Díez y Mirón de paz femenina (2004), o el enfoque de Paz Imperfecta (Muñoz: 2001), que como se ha dicho, servirá, a modo de antesala, de la paz transformadora.

El planteamiento de Muñoz se aleja de la concepción según la cual se considera la paz como ausencia de conflicto (y, por tanto, más relacionado con la violencia que con la propia paz), reafirmando el sentido de la paz por sí misma, reconociendo los fenómenos presentes en las acciones de los seres humanos capaces de crear paz, vinculando la paz a la cultura, las acciones, los pensamientos y los diálogos de los seres humanos.

${ }^{1}$ Según París y Martínez (2008), en los años cincuenta aparece el término resolución de conflictos, el cual hace referencia al modo de acabar con las consecuencias negativas que los mismo generan, sin prestar atención a otros aspectos como son la justicia social o la transformación de las causas que los originaron. 
De acuerdo con la concepción de la Paz Imperfecta, se entiende que la historia de la Humanidad ha sido interpretada poniendo el acento en aquellos aspectos relacionados con la violencia, la guerra o la lucha entre seres humanos, clases sociales, estados, gobiernos, naciones, etc., por encima de todas las manifestaciones pacíficas, colaboracionistas, cooperativas, solidarias y amistosas que también han contribuido, de manera decisiva, al desarrollo de las especie humana.

La teoría de la Paz Imperfecta plantea que la paz no ha de considerarse como un concepto dependiente y subordinado a la violencia y al conflicto. Desde esta perspectiva, se entiende que no sólo la paz negativa relaciona la paz con la violencia, sino que también desde la paz positiva se termina interpretando la paz en relación a una mayor o menor contundencia de las violencias estructurales. Para Francisco Muñoz, la paz se encuentra dotada de contenido en sí misma, sin necesidad de ser considerada en relación a la ausencia o presencia de guerra, o de violencias estructurales. De ahí el uso del adjetivo imperfecta, que hace referencia a que la paz es un proceso siempre inacabado creado por los seres humanos en cualquier ámbito o contexto. Incluso en el marco de las más terribles confrontaciones bélicas (Muñoz, 2004).

En este sentido, todas las experiencias pacíficas, construidas por los sujetos en sus acciones relacionales cotidianas, como "condición de posibilidad de las relaciones humanas y, a la vez, horizonte a conseguir" (Muñoz, 2001: 73), constituyen un ingente material de análisis, normalmente ajeno a su estudio dado el foco que tradicionalmente los científicos sociales han puesto en los aspectos violentológicos, a los que normalmente se ha tenido a bien reconocer bajo el nombre de conflicto, siendo la teoría del conflicto la encargada de su análisis. En contraste, esta propuesta defiende el reconocimiento de todas las experiencias de paz, independientemente de la existencia o no de violencias, que han contribuido a la continuidad de la especie humana.

Este enfoque, al desligar de la violencia la concepción de la paz, permite abordar, con mayor profusión, además de las consideraciones relacionadas con la violencia estructural y la atención de las necesidades básicas propuestas por Galtung (y anteriormente por Burton), el complejo universo de la convivencia humana.

Desde la perspectiva de la Paz Imperfecta, la paz se concibe como un proceso vivo creado por los propios sujetos. Ello nos permite relacionar esta corriente de pensamiento, con un enfoque sociopráxico: paz como constructo humano en constante transformación, fruto de las acciones humanas y las compatibilizaciones de sentidos que los seres humanos inferimos a las prácticas y acciones que realizamos o/y experimentamos, y que forjan diferentes convivencias en un determinado espacio de cohabitación o relacional. 
Recogiendo las aportaciones de la paz positiva y la paz imperfecta, desde el enfoque sociopráxico, es posible concebir la paz transformadora, como un proceso vital en constante búsqueda de equilibrio, dada la necesidad de compatibilizar las realidades que los sistemas humanos tenemos. Entendiéndose la Paz como un armónico desequilibrio del sistema, tal y como se explicará seguidamente.

\section{CONCEPTUALIZACIÓN DE LA PAZ DESDE LA SOCIOPRÁXICA NATU- RALEZA HUMANA: LA PAZ TRANSFORMADORA}

El ser humano es un sistema de observación práxico (que se transforma en la acción de transformar), organizacionalmente e informacionalmente cerrado y energéticamente abierto (receptor de estímulos y emisor de energíaª ${ }^{2}$, autopoiético (se autocrea) (Maturana y Varela, 1990), no trivial (capaz de innovar en las respuestas) (Foerster, 1991), y reflexivo (capaz de representarse a sí mismo y a otros sistemas observadores observando lo que él observa), que al acoplarse perceptivomotoramente con el medio y con quienes en él habitan recepciona estímulos a los que debe inferir su particular sentido, de acuerdo con la cultura hologramática insertada en su estructura organizativa. Sentidos que ha de procurarar compatibilizar en las redes en las que participa para su supervivencia, pues, para que el sistema funcione y pueda sobrevivir, precisa compatibilizar las realidades construidas. Esto es, las realidades, como se ha dicho, son construidas, pero no son caprichosas, pues, como asimismo se dice, se han de compatibilizar en las redes en las que se participa si se quiere seguir viviendo.

Las constantes entradas de estímulos provocan perturbaciones en el sistema, que impelen a la estructura organizativa del mismo a proceder a su clausura organizacional, generando orden interno, a la par que respuestas que generan desorden en el medio, autocreándose en el proceso. Los sistemas energéticamente abiertos, como somos los seres humanos, al mantener relaciones con el medio, se hallan inmersos en procesos entrópicos de desorden, encontrándose en un estado de no equilibrio; siendo, precisamente, a partir de ese desequilibrio como se genera, el orden, la estabilidad. Es los que Prigogine y Stengers denominan estructuras disipativas, esto es, estructuras que se forman a partir del proceso entrópico de una complejidad indeterminada para construir -a partir de ello, precisamente- un orden (de complejidad determinada o determinable) (Prigogine y Stengers, 1985). Dicho de manera coloquial, la

\footnotetext{
${ }^{2}$ Haciendo uso de la distinción de Bertalanffy entre sistemas cerrados, aquellos en los que ni entra ni sale energía o materia, y sistemas abiertos, aquellos en los que existe un constante intercambio con el medio (Bertalanffy, 1978)
} 
interacción genera desequilibrio al sistema, el cual le llevará a emitir una respuesta con la que lograr el equilibrio, pero es precisamente esa respuesta la que al alterar el medio producirá un efecto que volverá a propiciar un desequilibrio en el sistema, que obligará a emitir una respuesta que... Y así sucesivamente.

El sistema al emistir respuestas con las atender sus propias necesidades, o al reconfigurar las necesidades o la propia organización del propio sistema, logrará estabilizarse, encontrándose éste en un estado de "desequilibrio armónico". Esto es, en paz, o estable, dentro del perrmanente desequlibrio propio de la estructuras dispipativas.

La propuesta de paz transformadora, siguiendo este planteamieno, se concibe como armónicos desequilibrios de sistema, entre sistemas y redes, al considerar que es el marco del proceso de compatibilización de realidades construidas, donde emerge toda realidad social, y, por ende, tanto la paz como el conflicto.

La concepción de la Paz, desde una perspectiva transformadora, trasciende los límites de la paz positiva (y, obviamente, de la paz negativa), pues no se define en relación a la ausencia de confrontaciones bélicas o la, mayor o menor, influencia de las violencias directas, culturales o estructurales que plantea Galtung. La paz se relaciona con la convivencia en los ámbitos micro, meso y macro, y se entiende como un proceso vital de transformación de modelos de convivencia que inhiben o violentan la atención de las necesidades del conjunto de la población en modelos sinérgicos de atención integral de las mismas ${ }^{3}$. Se conceptualiza, por tanto, como un proceso que plantea cambios de las necesidades que cada ser humano construye, de los medios y modos de atenderlas, de las prácticas que realiza, así como de las redes de relaciones que cada uno y cada cual contrae en la vida sociocomunitaria.

Así, pues, la paz transformadora se entiende como el proceso vital en el que las perturbaciones internas del sistema se regulan buscando atender las necesidades del mismo, produciendo acciones-respuestas cuya recepción por parte de los demás sistemas producen respuestas (que, a su vez, serán valoradas) como no violadoras, inhibidoras o pseudosatisfactoras, sino sinérgicas y recursivas para la atención de sus necesidades. La multiplicidad, en el tiempo,

${ }^{3}$ Según Max-Neeff, Elizalde, y Hopenhayn (1986) hay cinco satisfactores o modos de atender las necesidades: a) Violadores y destructores: el modo que tienen estos satisfactores de atender una necesidad de uno es a costa de impedir o dificultar que puedan atenderse otra necesidad y que otros seres puedan también atender las suyas; b) Pseudosatisafactores son los que crean una falsa sensación de haber cubierto una necesidad; c) Inhibidores: esto atienden en demasía una necesidad impidiendo o obstaculizando que se puedan atender otras; d) Singulares, son los que sólo atienden una sola necesidad sin tener en cuenta la suerte que pueden correr las demás; e) Sinérgicos son los que atienden una necesidad estimulando y contribuyendo a que sean atendidas las demás necesidades. 
de respuestas-acciones y valoraciones, por parte de los diferentes sistemas, y la vinculación de sus redes, es parte constituyente del proceso de paz, siendo, por tanto, en el marco relacional-convivencial donde ésta (de igual modo que el conflicto) juega su papel protagonista. La producción y reproducción de convivencias más sinérgicas y recursivas de la paz o más violentas, dependerá de los cálculos, respuestas-acciones y valoraciones que sean capaces de operativizar los sujetos y sus redes, en sintonía con la atención de sus propias necesidades y las de los demás.

Esta conceptualización de la paz, supone tener en cuenta el medio y modo que tenemos los seres humanos de atender nuestras necesidades en los diferentes espacios de cohabitación e interrelación humana. En consecuencia, por un lado, se entiende la paz como un constructo dotado de valor en sí mismo, que debe ser desarrollado mediante el fomento de espacios de intercambio y relación que favorezcan la atención de las necesidades de unos y otros sistemas sinérgicamente, y, por otro lado, se considera necesario fomentar el desarrollo de un proceso de transformación de los conflictos, propiciando para ello, no sólo la participación y segunda reflexión de las partes contendientes, sino también de los diferentes sujetos y redes, que enredados, conforman el conflicto y cohabitan (o se relacionan) en el ámbito en el que tiene lugar.

\section{EL CONFLICTO SOCIAL DESDE LA PERSPECTIVA DE LA PAZ TRANS- FORMADORA}

De acuerdo con la concepción de la paz transformadora, se entiende que el sistema entra en crisis, cuando las perturbaciones inhiben al sistema de la posibilidad de atender sus necesidades, cuando no se procede a la redefinición del propio sistema de necesidades, o, cuando, aún procediendo a dicha redefinición, no se reorganiza el sistema.

Toda perturbación y su correspondiente valoración, tanto cuando encaja con el modo y el medio de atender las necesidades y demandas definidas por el sistema como cuando no es así, impele al sistema a su clausura organizacional en busca de su equilibrio interno. Si bien, se considera que únicamente cuando las acciones emprendidas por el sistema no logran atender sus necesidades y demandas, se estaría ante la posibilidad de la aparición de una crisis del sistema.

Esto implica que no toda entrada al sistema genere crisis, ni mucho menos, conflictos. Ante una situación potencialmente causante de crisis, el sistema deberá buscar nuevamente el modo de atender sus necesidades emitiendo nuevas respuestas, re-definiendo su propio sistema de necesidades, o bien, autoprogramándose. Si el sistema no lograra gestionar exitosamente su crisis interna, la profundización de su bloqueo generaría la muerte del mismo. La capacidad 
de gestionar las crisis, que todos los sistemas humanos poseemos, parte de la capacidad valorativa-creativa (o, si se quiere, habilidad transductora) propia del ser humano.

De este modo, se entiende que no se puede hablar de conflicto cuando es un mismo sujeto el que debate en su interioridad, pues de ser así, cualquier acto humano podría ser catalogado como conflictivo, incluso el respirar, y, por tanto, carecería de sentido decir qué se entiende por conflicto. Cuando un ser humano duda, o decide entre varias opciones, está realizando un ejercicio decisorio complejo, pero no hay conflicto. Cuando un determinado ejercicio termina sumiéndolo en un terrible dilema tal que le provoca una perturbación capaz de producir una crisis en su sistema, deberá hablarse de crisis sistémica, pero no de conflicto.

De la misma forma, la existencia de confrontaciones interpersonales en un espacio de cohabitación puede generar un conflicto social, sin embargo, no se considera que de todo problema interpersonal surja necesariamente un conflicto social. Si bien, la existencia de confrontaciones o problemas interpersonales es capaz, por sí misma, de impactar en la convivencia grupal de un modo más o menos relevante, en función de múltiples variables. Las confrontaciones o problemáticas interpersonales pueden ser germen de un conflicto y elemento de un conflicto social, pero no un conflicto en sí mismo.

En este sentido, se ha de apuntar que será la capacidad autoorganizativa y reflexiva no trivial del sistema humano, la que posibilitará transformar las crisis que podamos sufrir en nuevas energías capaces de atender las necesidades del propio sistema. La capacidad trasductora de inventar, articulando lo nuevo en lo conocido, que los sistemas humanos poseemos, nos posibilita cambiar malestares por armónicas relaciones convivenciales, y, también, a la inversa.

Del mismo modo que las crisis sistémicas suceden en el interior de los sistemas humanos, no siendo pertinente hablar de conflictos internos -sino justamente, de perturbaciones o/y crisis sistémicas-, cuando se hace referencia al ámbito social, es preciso referirse a problemáticas interpersonales, malestares, insatisfacciones y conflictos sociales. La relación existente entre crisis y conflicto no necesariamente es correlativa, pues no necesariamente de toda crisis se desprende un conflicto, si bien una crisis, podría ser capaz de generar un conflicto social, al igual que lo puede generar una confrontación interpersonal. Para que ello ocurra, será preciso que se cumplan una serie de requisitos, de acuerdo con la construida definición del conflicto que así se presenta:

El conflicto social es una realidad socialmente cristalizada. Socialmente compatibilizada, consistente en una relación entre sistemas humanos, en la que los efectos de las acciones de un o unos sistemas generan malestar en otros siste- 
mas, considerando los sistemas afectados que aquellos sistemas a los que atribuyen dichas acciones emprendidas, saben que las mismas (las acciones emprendidas) generan malestar en los primeros sistemas, impeliendo a éstos a emitir respuestas, cuya valoración interna por parte del segundo o segundos sistemas, genera algún grado de inquietud y/o malestar en el mismo o los mismos. Encadenándose multiplicidad de acciones y valoraciones entre uno o unos y otro $\mathrm{u}$ otros sistemas que perpetúan en el tiempo el malestar, las inquietudes o insatisfacciones, quedando implicadas en este proceso diversas redes socioculturales, y, por tanto, constituyendo una relación entre sistemas y redes capaz de producir y reproducir modelos convivenciales inhibidores, pseudosatisfactores o violadores mediante los que atender las necesidades humanas. Siendo, a su vez, los conflictos sociales y los modelos convivenciales producidos, causa y efecto al mismo tiempo de su propia producción y reproducción.

Préstese atención a los siguientes componentes de la definición:

1) Deben producirse acciones humanas. Lo que implica que deberá achacársele a otro sistema humano el malestar que el sistema padezca. Si, por ejemplo, es un perro quien muerde a un ser humano, es probable que el mordisco le genere un malestar terrible, pero nunca un conflicto con el perro. Si acaso, se podría llegar a generar un conflicto con el amo del perro, si se cumplieran otros requisitos.

Asimismo, un sistema no humano, jamás podría constituir un conflicto, dado que incluso admitiendo la posibilidad de que, por ejemplo, dicho perro pudiera atribuir a algún ser humano sus malestares, jamás podría llegar a considerar que lo que él ve (el perro), otros también lo ven. El perro, como el ser humano, es un sistema abierto pero, a diferencia del este último, no es reflexivo. Por tanto, incapaz de ver a otros viendo lo que él ve. De la misma forma, se entiende que no es posible hablar de conflicto en relación a un objeto inanimado.

2) Se dice realidad socialmente cristalizada no realidad compartida, esto es, no se trata de una misma realidad percibida por diferentes actores sino que los sentidos inferidos a las acciones perceptivomotoras que se desprenden de las respectivas y singulares percepciones sobre la realidad llamada conflicto social, encajan entre sí.

El conflicto social, como cualquier otra realidad social, requiere ser observada como realidad externa y sustantiva. Es decir, requiere constituirse como una realidad cristalizada. Ello es posible, gracias a la capacidad reflexiva del ser humano y el efecto de realidad objetiva que se produce al compatibilizar realidades. Al ser toda realidad personal e intrasnferible, construida por cada sujeto, para que emerja una una realidad social externa y sustantiva, será preciso que la realidad personal encaje con la de otros sujetos, haciéndose compatibles las realidades construidas. 
Los seres humanos nos representamos representando la realidad que construimos, y, compatibilizándola con otros sujetos, somos capaces de cistalizar realidades sociales. Por tanto, para que exista conflicto social, es necesario que el sentido inferido atribuido a las acciones emprendidas por determinados sistemas humanos que generan malestar, sea configurado como una externa realidad grupalmente compatibilizada y cristalizada con otros sistemas reflexivos.

Y en este sentido, cabe prestar atención a las siguientes cuestiones:

2') Como se ha anotado, si no se compatibiliza el sentido inferido con otras realidades grupales construyendo una realidad externa y objetiva, no se estará ante realidad social alguna, y, por consiguiente, ante ningún conflicto social.

2") Existirán tantos conflictos sociales como realidades cristalizadas al respecto sean producidas. La compleja realidad llamada conflicto social, implica que diferentes sistemas produzcan diferentes valoraciones respecto de las causas que generan sus insatisfacciones, pudiendo atribuirlas a diferentes comportamientos o acciones humanas, pero si ninguna de éstas valoraciones logra ser cristalizada socialmente, tampoco se estará ante un conflicto social. Es decir, para que estemos en presencia de un conflicto social, es necesario generar ciertos acuerdos respecto de la veracidad del hecho que el sistema insatisfecho compatibiliza con otros sistemas. De lo contrario, podría ocurrir como en el siguiente ejemplo:

Imagínese que a una máquina de producción de una cadena de montaje deja de entrarle energía, creando en ella una crisis en su organización interna, y deteniéndose. E imagínese que el Jefe de producción, achaca el problema a una acción de sabotaje de unos empleados, pero al compatibilizar dicho sentido inferido con sus superiores e incluso la junta de accionistas de la empresa no le otorga la más mínima credibilidad. En este caso, se podría decir que el jefe ha sufrido un episodio paranoico, incluso pudiendo ser acertada la valoración. Pues al no ser posible cotejar la realiadad construida con ninguna realidad externa, sino con las construcciones de realidades de cada sujeto y su compatibilización; al no otorgarle ningún otro sistema reflexivo verosimilitud alguna al sabotaje, no es posible hablar de conflicto social (o sociolaboral, si se quiere especificar el ámbito en el que se ha producido), sino únicamente de malestares del sistema llamado Jefe de Producción, que podrían conducirlo a una crisis psicótica, de no ser capaz de gestionar adecuadamente las valoraciones de las respuestas efectuadas por los sistemas con quien el Jefe de producción compatibilizaba su sentido inferido.

Asimismo, obsérvese que al ser el conflicto una realidad social cristalizada, cabría la posibilidad de entender que una situación de insatisfacción en unos sistemas humanos, fuera producto de unas valoraciones, $\mathrm{u}$ otras, pudiendo 
producirse, tras la pertinente compatibilización de sentidos inferidos, diferentes realidades cristalizadas, y, por tanto, diferentes conflictos sociales. Por ejemplo, allí donde unos sistemas humanos constituidos por "obreros de la construcción", cristalizan la realidad social "conflicto laboral" constituida por la relación entre directivos de empresa y obreros, otros "obreros de la construcción", pudieran hacer lo propio cristalizando la realidad social "conflicto migracional-laboral", donde la población inmigrante podría ser parte constituyente del mismo, al -erronéamente- achacar a ella los males provocados por la asunción de puestos de trabajo con menores prestaciones y/o garantías sociales. Nótese que sendos conflictos sociales son capaces de coexistir en el tiempo, produciendo acciones-respuestas y valoraciones sobre dichas acciones-respuestas diferentes, así como implicando a diferentes redes y generando diferentes efectos convivenciales.

3) No son las acciones (inicialmente realizadas o como respuestas a las recepcionadas) las que generan el malestar, inquietud o insatisfacción en el sistema, sino la valoración interna que de las acciones o respuestas recepcionadas realiza el sistema.

Préstese atención, pues esta conceptualización del conflicto, supone asumir que las estructuras, poderes políticos o económicos, por sí solos, no son los productores de conflictos sociales, aunque en buena parte suministren los estímulos para que se produzcan; sino que su emergencia, depende de la inferencia de sentido que los sistemas receptores de estímulos realizan de acuerdo con las propias necesidades y cultura hologramática, mediante la cual juzgan como causantes de sus malestares a determinadas acciones emitidas por unos sistemas humanos, u otros. Ello implica que los malestares que unas acciones producirían en otros sistemas o/y redes, no sean producto de la acción sin más, sino de la inferencia de sentido efectuada por el receptor.

4) Se dice que unos sistemas saben que otro u otros sistemas saben que sus acciones generan malestar, no que necesariamente el otro u otros sistemas sepan, y, por tanto, que

4') no necesariamente ha de haber intención en generar malestar por las acciones emprendidas; pues,

4") el sistema al que se le hace responsable del malestar puede no saber que sus acciones generan malestar, y

4"') el sistema al que se le hace responsable del malestar no necesariamente es el responsable del malestar padecido por el otro sistema.

Es necesario que el sistema humano que ha valorado las acciones de otro sistema humano como las causantes de su malestar (o insatisfacción), ha de ver que el sistema causante es capaz de ver lo que él ve. Obsérvese que se dice 
que el sistema ha de ver que el sistema causante es capaz de ver, y no que el supuesto sistema causante, en efecto, vea lo que él ve. Complementariamente a este requisito, vale la pena apuntar que no es necesaria la atribución de intencionalidad en el acto.

Para producirse conflicto social, al menos, un sistema humano debe atribuir a las acciones de otros seres humanos la responsabilidad sobre sus malestares, inquietudes o insatisfacciones, asumiendo que otros seres humanos son capaces de ver lo que él ve. Y compatibilizándolo con otros seres humanos, se construya una realidad social cristalizada que provoque la concatenación de valoraciones y acciones entre sistemas y redes.

Por esto, se dice que para poder hablar de conflicto, el sistema tiene que atribuir a los sistemas causantes de sus insatisfacciones, la responsabilidad de ser capaces de ver que podrían ser los causantes de sus malestares o inquietudes ${ }^{4}$. Esto no significa, como se advertía, que el sistema reflexivo insatisfecho vea que el sistema presuntamente causante coincida en su diagnóstico, sino que al compatibilizar con otros sistemas, ve, $-\mathrm{o}$, dicho con mayor precisión, no repara en que su percepción es su percepción y no la realidad para ser percibida. O sea, no repara en que la realidad percibida es la suya y no una realidad externa objetiva para ser percibida- que otros también consideran que determinadas acciones humanas son las causantes o generadoras de su malestar.

Por ejemplo, se podría hablar de un conflicto social cuando los adultos se quejan del ruido que hacen los jóvenes por las noches, aunque los jóvenes no estén de acuerdo con la valoración de los adultos e incluso no sepan que su acción genera malestar, siempre y cuando los adultos compatibilizaran con otros sistemas grupales la visión de que los jóvenes saben que los adultos se quejan de que los jóvenes hacen ruido por las noches y les atribuyen a ellos ser los causantes de dichas perturbaciones; produciéndose sucesivas acciones y valoraciones dilatadas en el tiempo, implicando a otros sistemas y redes.

5) Se produce una cierta continuidad temporal, y una sucesiva concatenación de acciones y valoraciones entre sistemas, quedando implicadas sucesivas redes socioculturales, impactando en la realidad convivencial.

Es necesario que, de alguna manera, se produzca una concatenación de acciones-respuestas y valoraciones entre sistemas, implicando a diferentes redes. Y no necesariamente de forma bidireccional entre el sistema insatisfecho, sus redes y el valorado por éste como causante de sus insatisfacciones y sus respectivas redes.

${ }^{4} \mathrm{Y}$ como tales sistemas reflexivos, tienen la posibilidad de dudar entre la subjetividad y la objetividad, y ello implica tener dudas sobre si lo que se ve es lo que hay para ser visto o es lo que se ve porque uno y sólo uno es quien lo ve. 
Obsérvese que, por tanto, se entiende que:

5') Es preciso que se emitan acciones-respuestas y las consiguientes valoraciones de las mismas, entre sistemas y redes, si bien no es necesario que dichas acciones-respuestas impliquen el uso de la violencia, la agresión o la confrontación directa. La existencia de acciones-respuestas de carácter violento o confrontativo, no son imprescindibles para constituir un conflicto social.

5") La multiplicidad de acciones-respuestas emitidas y recepcionadas, serán dirigidas por parte de los diferentes sistemas y redes de modo que atiendan a sus propias necesidades, respondiendo a los cálculos que éstos hagan de acuerdo con su propia cultura hologramática o su compatibilizada cultura, respectivamente.

5"') Las acciones-respuestas emitidas por los sistemas y redes obedece a un modelo de relación multidireccional y multiplicador, no únicamente a un modelo de relación reticular bidireccional. Al ser el conflicto una realidad reticular en la que se encuentran vinculadas diferentes redes, las respuestasacciones que se produzcan afectarán no sólo a aquellas redes o sistemas valorados como causantes de los malestares o insatisfacciones, sino también puede implicar a multiplicidad de sistemas y redes.

En este sentido, es preciso tener en cuenta que, por un lado, todo sistema hace responsable a otros sistema de su malestar, independientemente de que así sea, no existiendo forma de comprobar que el sistema acusado de ser el causante de determinados malestares, sea el realmente causante de tales malestares; por otro, la sucesiva implicación de una red y/o sistema en un conflicto, se produce obedeciendo a la atención de las propias necesidades ${ }^{5}$, produciéndose una realidad relacional enredada; y, por último, que el efecto multiplicador de redes y relaciones interreticulares e intrareticulares partícipes en un conflicto, supone una concatenación capaz de trascender el germen inicial, implicando a nuevos sistemas y redes, nuevas acciones-respuestas y valoraciones, potencialmente infinita, haciendo maleable la estructura del conflicto.

Por tanto, será preciso que se produzcan multiplicidad de acciones-respuestas, implicando a diferentes sistemas y redes. Esta concatenación de acciones-respuestas, generará un proceso de intervinculación reticular al que de manera dilatada en el tiempo se incorporarán (o desagregarán) diferentes

${ }^{5}$ Pero ello no significa que los sistemas-redes implicados en el conflicto, lo hagan felizmente y de manera voluntaria. Por ejemplo, la responsabilidad de defender el honor familiar que recae en los hombres de las familias gitanas, supone que ante cualquier deshonra, deban intervenir en favor de la propia familia. Priorizando la atención de la necesidad de mantener la honorabilidad, auque ello suponga poner su vida en peligro. 
sistemas y redes. El modo de actuar de los diferentes sistemas y redes en el marco del conflicto social, y las acciones-respuestas y valoraciones que de éstas se hagan, dependerán de los cálculos, lógicas y estrategias que las redes y sistemas pongan en práctica de acuerdo con la atención de sus propias necesidades, siendo, por tanto, imprescindible hacer partícipe a los sistemas y redes en el análisis y la construcción de alternativas para la transformación del conflicto.

Recuperando el ejemplo citado, para hablar de la realidad cristalizada "conflicto laboral", sería preciso que en la relación entre las redes de los "empresarios" y los "obreros", el "empresariado", ante la respuesta de los "obreros", mantuviera o reafirmara la posición acusada de ser la causante de las insatisfacciones de los sistemas "obreros de la construcción". Por el contrario, esto es, si la empresa, o hablando con más propiedad, si los directivos de la empresa u otros sistemas dotados de responsabilidad al respecto (léase Estado, administraciones públicas, sindicatos, medios de comunicación, etc.), emprendieran una acción correctora que pusiera fin al efecto valorado por los obreros como causante de sus malestares, no podríamos hablar de realidad cristalizada "conflicto laboral". Es decir, si la directiva de la empresa accediera a las solicitudes de los sistemas "obreros de la construcción", la realidad cristalizada "conflicto laboral" no hubiera tenido lugar, pues la relación entre empresa y trabajadores, no hubiera pasado de malestar en el sistema-red "obreros de la construcción".

Pero si, llegado el caso, la dirección de la empresa hiciera caso omiso a las reivindicaciones laborales presentadas por los obreros, manteniendo sus acciones de acuerdo con las lógicas y estrategias empresariales de atención de sus propias necesidades (maximilizando los beneficios, por ejemplo), esta sitaución, podría generar una nueva respuesta por parte de la red "obreros de la construcción" en forma de comunicado de prensa, acciones de prostesta diversa o incluso, la huelga. Ambas acciones, implicarían, la multiplicidad de nuevos sistemas y redes (y, por tanto, la extensión del conflicto y/o la emergencia de nuevos conflictos): la población que se ve afectada por la huelga, otras empresas relacionadas, el ministerio de trabajo, del interior, la policía, los medios de comunicación, etc. donde cada uno de ellos, se vería impelido a realizar una valoración, emitiendo una determinada respuesta.

El efecto multiplicador de todo conflicto social, es potencialmente capaz de forzar la desaparición o desdibujar el gérmen que desencadenó el malestar inicialmente. Ya que, el propio proceso dilatado en el tiempo de un conflicto social y los numerosos elementos que lo constituyen, podría propiciar la emergencia de nuevos conflictos. Del "conflicto laboral" podría emerger, por ejem- 
plo, un "conflicto político social", si las redes "obreros de la construcción" propician la participación de otras redes como la de los estudiantes, indignados, parados, inmigrantes, etc. e iniciasen acciones colectivas e individuales capaces de generar un proceso que implicaría a su vez la participación de nuevas redes, respuestas y valoraciones, por parte del gobierno, la patronal, etc.; así como unos nuevos efectos y consecuencias para la convivencia social.

Los impactos de los conflictos en la realidad convivencial, suponen la perpetuación del malestar, y la potencial reproducción de modelos convivenciales inhibidores, pseudosatisfactores o violadores de la atención de las necesidades de los sujetos.

Así, se entiende que son las apuestas por modelos inhibidores, violadores o pseudosatisfactores de la atención de las necesidades propias, elementos que favorecen la emergencia del conflicto social y la reproducción de modelos perpetuadores de convivencias no pacíficas.

La participación de diferentes redes en un conflicto, conllevará la apuesta por la implementación de estrategias y acciones con las que atender sus construidas necesidades. Ahora bien, ha de saberse que, si bien las redes son parte constituyente de los sujetos mediante las cuales se nutren de materia prima para la construcción de sus realidades, culturas y necesidades, la participación de los diferentes sujetos en las mismas constituidas redes sociales no será nunca igual, ni implicará la reproducción detallada de modos de proceder predeterminados, pues, en primer lugar, dentro de las redes sociales existen diferentes subredes, e incluso diferentes niveles de adscripción o identificación con sus principales postulados identitarios; y en segundo lugar, dada la inherente capacidad humana para construir transductivamente la propia realidad de acuerdo a la propia e intransferible cultura hologramática, se puede transformar los modos de dar atención a las necesidades de forma inhibidora o violadora, en otro tipo de fórmulas creativas sinérgicas de atender las propias y ajenas necesidades.

De ahí, la necesidad de hacer partícipe a la población en la transformación de conflictos, según el modelo de Construcción Participada de Convivencias Pacíficas que se describe más adelante. Esto es, hacer partícipes a los diferentes sistemas y redes constituyentes del conflicto en una reflexión colectiva respecto de las acciones-respuestas, valoraciones de éstas, efectos e implicaciones que dicho conflicto supone en sus espacios de cohabitación o interrelación humana, con la finalidad de construir e implementar nuevas acciones y estrategias capaces de transformar los efectos que generan insatisfacción, en fórmulas sinérgicas y recursivas para la atención de las necesidades del conjunto de sistemas humanos en el ámbito micro, meso y macro. 


\section{CONTEXTOS Y LÓGICAS COMPLEJAS A TENER EN CUENTA EN LA PLANIFICACIÓN DE LOS PROCESOS PARTICIPATIVOS DIRIGIDOS A MEJORAR LA CONVIVENCIA}

Manuel Montañés (2009) identifica cuatro contextos que forman parte del esquema mediante el cual cada sujeto infiere sentido, y que aquí, se interpretan como parte de la dimensión contextual. A saber: el contexto gramatical, el psíquico, el situacional y el socioeconómico-histórico-cultural.

El contexto gramatical, hace referencia a cómo se debe hablar y escribir la lengua que el sujeto compatibiliza, pues aunque no sea necesario conocer los significados cristalizados de todos los significantes, el sujeto, impelido por su necesidad de hacerse entender y entenderse con el resto (de inferir sentido y de que el resto infiera sentido a lo que éste dice), precisará comunicarse formulando frases capaces de encajar con la semántica, morfología y sintaxis de las personas con las que interactúa. Y esta situación condicionará el modo en el que el sujeto proceda a construir conocimiento y transmitirlo. Los sentidos que se infieren están condicionados por las reglas y normas de la lengua que se compatibiliza; siendo las normas, las reglas prácticas de la cultura que se compatibiliza.

El contexto psíquico se refiere a los deseos, sentimientos y pasiones a los que cada sujeto se encuentra sujetado en el proceso de construcción de la realidad y relación transubjetiva.

Por su parte, el contexto situacional se refiere, precisamente, al contexto espacio-temporal donde se dan cita las relaciones y al modo en el que se pronuncian los estímulos. Un mismo estímulo recepcionado en diferentes momentos puede interpretarse de muy distintas formas, según el sujeto infiera su particular sentido, de la misma forma que según sea el tono de voz o la gesticulación con la que se pronuncie el estímulo. Es evidente que una gesticulación concreta a la hora de decir algo, o un determinado énfasis puede producir efectos en el proceso de inferir sentidos diferentes a aquellos que la misma frase pronunciada de forma relajada o en un contexto espacio-temporal diferente.

Respecto del contexto socioeconómico-histórico-cultural, éste se refiere a las redes de relaciones y status socioeconómico, de género, edad o hábitat convivencial donde el sujeto cohabita, así como al sistema de "creencias, valores, experiencias y expectativas que en cada una de estas redes se compatibilizan." (Montañés, 2009: 60-62)

Al estar obligados los seres humanos a compatibilizar las realidades en los espacios de cohabitación, responderemos práxicamente de diferente forma, consolidando prácticas sociales en redes sociales, y, por tanto, produciendo, reproduciendo y transformando diferentes conflictos y modelos convivenciales. 
Analizar colectivamente la dimensión Contextual a la que se hace referencia, implica tener en cuenta los contextos mencionados, y supone detectar qué problemáticas sociales existen en los diversos espacios de cohabitación, qué malestares e inquietudes en qué sujetos y qué redes, cómo se relacionan entre e intraredes, tener en cuenta los deseos, sentimientos y pasiones, de qué modo se regulan los conflictos, dónde se producen, cuándo y porqué, qué normatividades están presentes en el entorno sociocultural, cómo se ejercen los poderes, las vivencias históricas de cada grupo, los usos y costumbres, y las cuestiones particulares susceptibles de provocar emergencia de conflictos.

En este sentido Lederach (1997), siguiendo el paradigma anidado de los enfoques del conflicto propuesto por Dugan (1996), apuesta, por un modelo de regulación de los conflictos que parte de la vinculación del sujeto en el análisis de la cuestión en conflicto para lograr su transformación, capaz de "considerar las cuestiones más limitadas y los aspectos sistémicos más amplios de la resolución del conflicto y la construcción de la paz" (Lederach, 1997).

Desde el paradigma que se defiende en este artículo, se propone dar un paso más, pues al considerar que los aspectos sistémicos de un conflicto, de igual modo que las cuestiones más limitadas, son construidos por los sujetos, es preciso articular un proceso de investigación-acción-participativa, capaz de promover las reflexiones de los diferentes sujetos en y entre sus redes, respecto de las propias dimensiones contextuales y estructuras reticulares que, de un modo u otro, influyen en todo el proceso de compatibilización para formular e implementar modelos convivenciales.

El análisis reflexivo sobre la dimensión contextual del conflicto, hace referencia a los elementos que constituyen los contextos psíquico, situacional y socioeconómico-histórico-cultural que forman parte de la cultura hologramática mediante la que los seres humanos infieren sentido, y, por tanto, construyen convivencias. En consecuencia, el análisis deberá contemplar la detección y valoración colectiva de los sistemas-redes y relaciones existentes, las culturas y subculturas así como los usos y costumbres, la cuestión de la que habla Lederach (1997), o las molestias, prácticas, inquietudes e insatisfacciones de los sistemas (como gérmenes potencialmente productores de conflictos sociales), las problemáticas existentes y las estructuras (o aspectos sistémicos sobre los que reflexiona Dugan (1996)), los poderes que forman parte de la convivencia sociocomunitaria, las vivencias históricas, el ámbito situacional y las normas vigentes formales e informales presentes en los espacios de cohabitación sociocultural.

Las problemáticas y los conflictos sociales deben ser analizados desde una perspectiva "lógica compleja, dialógica, hologramática y recursiva" (Morin, 
1995: 106) que rompa con el modelo clásico bipolar. En un conflicto social, no solamente es posible distinguir entre "buenos y malos", o entre "agresores y agredidos". Los posicionamientos y acciones de los sujetos van mucho más allá de tal simplificadora categorización.

Los sujetos y sus redes, proceden de acuerdo a una serie de lógicas que implican diferentes posicionamientos discursivos y prácticas sociales. Lógicas que insertadas en el esquema de producción de conocimiento de los sujetos, los impelen a realizar prácticas sociales en busca de la atención de sus propias necesidades. El quid de la cuestión, está en dar con las lógicas que provocan unas u otras posiciones discursivas, estrategias y acciones que las diferentes redes practican en cada momento, y reflexionar participativamente para transformarlas.

La superación de las dicotomías clásicas merced a la lógica compleja, permite estudiar las problemáticas desde perspectivas no bivalentes. De hecho, la multidimensionalidad del sujeto, es capaz de provocar acciones simultáneas aparentemente contradictorias, pero práxicamente complementarias. Los seres humanos, en su acción cotidiana, hacen uso de las estrategias de "compromiso", cuando se hace una cosa y su contraria vulnerando el principio del tercero excluido; "compartimentación", por la que se hace una cosa y otra diferente en distintos planos espacio-temporales anulando el principio de identidad; "oscilación", que rompe con el principio de no contradicción potenciando la paradoja de hacer una cosa haciendo lo contrario; e incluso "ilogización", capaz crear nuevas lógicas con las que formular problemas y el modo de resolverlos (Montañés, 2006: 304).

Planificar estrategias de manera participada para la transformación de los conflictos y la construcción de convivencias pacíficas, requiere tener en cuenta esta dimensión lógica-complejo-estratégica; pues para establecer consensos sobre las acciones a implementar con el propósito de alcanzar un determinado fin en un espacio de cohabitación concreto, previamente se debe conocer qué lógicas y estrategias guían los posicionamientos discursivos y las acciones de los sujetos y las redes que lo habitan.

En el juego social, cada actor, implementa sus estrategias, con el enfoque puesto en la atención de sus necesidades. Por lo que, de alguna manera, se podría sostener que los sujetos siguen estrategias tal y como Matus lo entiende: "una estrategia es un cálculo de interacción social que toma conciencia de la existencia del 'otro' para alcanzar el resultado perseguido [teniendo en cuenta a los otros jugadores y sus propias estrategias] para aumentar la probabilidad de éxito del resultado perseguido" (Matus, 2007: 380).

El propio Matus (2007) considera la planificación social dentro de un escenario que parte de una Gran Estrategia, en la que se deben definir los grandes 
horizontes finales, escenarios de futuro soñados/deseados, con el Juego Social, abierto e imprevisible en el que se dan cita las relaciones de cooperación y confrontación entre sujetos y redes, de acuerdo, precisamente, con las estrategias de cada sujeto y red: un espacio social cambiante y dinámico, de intereses y juegos de lógicas divergentes, donde las redes en las que los sujetos participan (y construyen en su acción de participar) se relacionan las unas con las otras, condicionándose las unas y las otras -sin ser posible controlar, prever o determinar qué y cómo sucederán las cosas-, pero siendo posible canalizar reflexiones colectivas orientadas en un determinado sentido.

Contemplar las estrategias de las redes y los actores para la planificación de acciones encaminadas a la transformación del conflicto requiere de la elaboración de un autodiagnóstico donde se plasmen las diferentes posiciones discursivas de las diferentes redes y las estrategias lógicas que las sustentan, además de dar cuenta de los diferentes estilos con los que los sistemas y las redes constituyen el conflicto, cumpliendo determinados roles: la cooperación/confrontación, la imposición, o el carácter negociador o mediador y los roles más activos o pasivos, observadores, confrontativos, etc. con los que actúan.

Este autodiagnóstico ha de ser considerado de acuerdo con la capacidad valorativa/creativa que atesora todo sistema humano.

En última instancia, la atención o no de las necesidades en un proceso de compatibilización de realidades, dependerá de la valoración interna que haga el sujeto de los estímulos que recepcione.

Esta dimensión valorativa-creativa del proceso de compatibilización de realidades hace referencia al modo en el que cada ser humano se autoorganiza en busca del equilibrio interno. Teniendo en cuenta la valoración del estímulo recepcionado como elemento favorecedor o no de la atención de las propias necesidades, así como la capacidad de creación y transformación de las propias necesidades y realidades para hacerlas encajar con las nuevas inferencias de sentido realizadas.

De esta manera, si la valoración que realiza un sujeto sobre los estímulos es entendida como favorecedora de la atención de ciertas necesidades, es evidente que de un modo u otro, lo habitual será que dicho sujeto, procure su reproducción. Si, por el contrario, los estímulos recepcionados son interpretados como elementos violadores o inhibidores de la atención de las propias necesidades, lo habitual será entonces que el sujeto trate de articular estrategias y acciones para hacer frente a dichas perturbaciones, como por ejemplo, podría ser huir, o luchar, o adaptarse a las circunstancias (que por otra parte, suele ser lo mayoritario). Decir "sí" y procurar hacer "no", o, apostar por fórmulas "si/no", o, "no/si". 
Tener en cuenta esta capacidad del sujeto para transformar y reconfigurar el propio sistema de necesidades, es tan importante como tener en cuenta las acciones implementadas por los sujetos para atender sus necesidades. La recreación del conjunto de necesidades para hacerlas encajar con las valoraciones realizadas a los estímulos recepcionados, se convierte en un modo habitual para la hacer frente a las perturbaciones del sistema; y esa capacidad, en forma de resiliencia, representa un elemento sobre el que es posible construir procesos de transformación individual y social.

Dicho de otro modo, no sólo interviene en el proceso de compatibilización de realidades la valoración que hace el sujeto de los estímulos que recepciona, sino también la capacidad de transformar el propio sistema de necesidades. Variando su priorización, transformando su contenido. De hecho, en ocasiones la autoorganización del sistema es de tal magnitud, que es capaz de vencer cualquier agresión (cualquier valoración realizada sobre un determinado estímulo, aparentemente violador de las necesidades del propio sistema), logrando re-construir nuevamente las necesidades para el equilibrio interno del sistema.

Esta capacidad de resiliencia, esto es, la capacidad que nace de la interacción, que permite a un ser humano, afrontar y superar los problemas y adversidades, construyendo sobre ellos respuestas creativas (Melillo y Ojeda, 2001), ha sido ampliamente estudiada por diferentes autores, proponiendo diversas definiciones y formas de intervención psicosocial sobre la misma. Mientras que para una persona puede ser una necesidad imperiosa atender determinada necesidad, para otros puede no serlo tanto. Por ejemplo, el concepto de dignidad es relativo, ya que una persona puede preferir morir antes que renunciar a sus ideales, y otra, puede rebajar sus principios si con ello logra mejorar, o no empeorar socioeconómicamente.

Además se ha de tener en cuenta no solo la dimensión instrumental sino la expresiva o de identificación grupal, que a toda realidad se le infiere, con mayor o menor grado, máxime en la actualidad en la que, como acertadamente sostiene Francisco Francés, hay un debilitamiento de los espacios tradicionales de sociabilidad formal, abriéndose camino nuevas formas de interacción social mucho más difusas, con vocaciones tanto generalistas como sectoriales, que vienen a ocupar el lugar de las vías tradicionales de participación. Y se trata de una sociabilidad que se desenvuelve no tanto a partir de las motivaciones racionales de los sujetos (sociabilidad instrumental), sino como respuesta de los sujetos para satisfacer necesidades de identidad (sociabilidad expresiva), construyéndose un universo de identidades complejas, fragmentadas pero articuladas (Francés, 2005: 103) 
Por su parte, la capacidad de reorganización del sistema de necesidades es tal, que, por ejemplo, existen personas que logran repriorizar su sistema de necesidades levantándose, en la misma cama del marido, tras la vigésima paliza del mes, construyendo estrategias vitales que les permiten no sólo sobrevivir resistiendo las enormes perturbaciones que su sistema sufre, sino atender necesidades mientras que construyen sus propias iniciativas de transformación de la situación.

Estas situaciones, en las que lógicamente intervienen muchísimos elementos, muestran claramente una dimensión a tener en cuenta en cualquier proceso de transformación de conflictos y problemáticas socioculturales. Las necesidades humanas constituyen creaciones personales e intransferibles, susceptibles de ser transformadas y (re)priorizadas en todo momento; razón por la cual, es posible que se reproduzcan comportamientos que consolidan prácticas sociales y las normalicen, de igual modo que es posible potenciar transformaciones desde la resiliencia, que impliquen cambios en las convivencias y en uno mismo (desde la reorganización del sistema de necesidades), contemplado la potenciación de diversos atributos, tales como la autoestima consciente, la introspección como el arte de preguntarse a sí mismo, la independencia para mantener distancia emocional y física sin caer en el asilamiento, la capacidad de relacionarse para establecer lazos e intimidad, la iniciativa para exigirse y ponerse a prueba, el humor para encontrar la comedia en la tragedia, la creatividad, el altruismo para extender el deseo personal de bienestar al resto de semejantes y la capacidad de pensamiento crítico para analizar críticamente las causas y responsabilidades de las adversidades que se sufren (Szarazgat el al, 2006).

Por ejemplo, la deplorable violencia de género es asumida por numerosas mujeres maltratadas como una realidad habitual, sobre la que suelen proceder reorganizando su propio sistema de necesidades. "La violencia contra la mujer está presente en todos los ámbitos sociales y económicos y está profundamente arraigado en las culturas de todo el mundo, hasta tal punto que millones de mujeres lo ven como un modo de vida", en palabras de Cate Johnson en un informe de Médicos del Mundo (2009). Un modo de vida producto de un proceso de compatibilización de realidades entre sistemas en el que ven atendidas sus necesidades ${ }^{6}$, bien a través de la valoración de la agresión como un hecho que aunque perturba al sistema, no lo bloquea, o, incluso, lo favorece (lo hace/hago por mi/tu bien), bien a través de la construcción de un sistema

\footnotetext{
${ }^{6}$ Aunque este proceso de compatibilización no produzca felicidad o placer alguno a los sistemas humanos, pues una cosa es atender necesidades y otra que ello proporcione mecesariamente felicidad. Téngase en cuenta que la atención de las necesidades se estrcucura en un marco de prioridades.
} 
de necesidades donde la violencia no viola la atención de aquellas priorizadas sobre otras (la protección, la compañía, el alimento... por ejemplo), por lo que es asumido como un modo de vida adecuado para estos sistemas.

La capacidad del ser humano para crearse y re-crearse a sí mismo, posibilita transformar las realidades convivenciales. Incluso aquellas en las que, como en el ejemplo mencionado, se vive con total normalidad la violencia de género, en ocasiones incluso negando la problemática dada la adecuación del sistema de necesidades de los sujetos al modo por el que se gestiona la relación.

La dimensión valorativa-creativa de los procesos de compatibilización de realidades cobra vital importancia a la hora de abordar procesos de transformación del conflicto y construcción de paz: si son los sujetos quienes crean y recrean sus realidades y situaciones de confrontación, creándose y re-creándose a sí mismos, son también los mismos sujetos quienes pueden crear y re-crear realidades y situaciones de atención sinérgica de las necesidades de unos y otros, a pesar, incluso, de la práctica de determinadas acciones realizadas por otros sistemas humanos ${ }^{7}$.

En este sentido, parece claro que prescindir del elemento valorador-creativo es poco acertado, aunque sea el modo de proceder más habitual en las negociaciones de paz clásicas, sobre la base de reuniones de alto nivel entre diplomáticos, jefes de Estado o/y líderes políticos. La exclusión de los sujetos que se encuentran en primera línea de fuego (y, normalmente también, en primera línea de miseria) de un proceso de transformación de conflictos para la construcción de paz, suele terminar en sonoro fracaso, por no considerar las dimensiones valorativas-creativas de los sujetos que forman parte del conflicto como víctimas y verdugos al mismo tiempo. Como partícipes de las convivencias violentas, y víctimas de su reproducción...

La capacidad del sujeto de transformar la realidad en el mismo proceso de crear la realidad, valorando los estímulos que recepciona, posibilita iniciar nuevos procesos reflexivos destinados a transformar la situación sociocultural establecida. Es posible cambiar las respuestas o su modo de responder, o modificar las necesidades o las prioridades o el modo de atenderlas. En consecuencia, contar con los sujetos en un proceso cuya finalidad última es la construcción de más y mejores espacios de convivencias pacíficas, es un requisito imprescindible.

${ }^{7}$ Otra cuestión es la referida a la pertinencia o no de promover reflexiones y prácticas en dichas convivencias, susceptibles de promover transformaciones en el modelo relacional establecido, sin haber sido "llamados para ello". Debate, por cierto, a todas luces estéril, alimentado con oscuras intencionalidades. 


\section{EL CICLO DEL CONFLICTO Y LA PAZ DESDE EL ENFOQUE SOCIOPRÁ- XICO DE LA PAZ TRANSFORMADORA}

De acuerdo con el enfoque propuesto, tanto la emergencia de conflictos sociales, como de espacios convivenciales pacíficos, obedecería al siguiente ciclo:

1. Acoplamientos perceptivomotores entre sistemas reflexivos.

2. Recepción de estímulos, valoración e inferencia de sentido de acuerdo al principio de reflexividad: ver al mismo tiempo que se construye lo que se ve, y, por ende, viendo a otro que ven lo que uno ve, emergiendo, de esta forma, el efecto de externa realidad objetiva.

3. Clausura organizacional y autocreación práxica del sistema. Emisión de respuesta.

4. Proceso de compatibilización de realidades (construcción de sintonías con otros sistemas reflexivos de acuerdo al sentido inferido y difusión).

5. Concatenación de acciones-respuestas y valoraciones que implican a nuevas redes.

- Resistencias, escalada y multiplicidad de acciones-respuestas que profundizan o mantienen los malestares y/o inquietudes de los sistemas-redes, enredando a nuevos sistemas y redes: emergencia de conflicto social; y/o,

- los sistemas-redes ven cubiertas sus necesidades sin producirse malestares, o, incluso, sinérgica y recursivamente potenciándose el bienestar de los sistemas-red y la atención de las necesidades de otros sistemas-red: emergencia de espacios de convivencias pacíficas, desequilibrios armónicos, paz.

- O bien, se produce una realidad cristalizada conflictiva, transformándose ésta mediante la redefinición y reorganización del sistema de necesidades y/o la repriorización de necesidades por parte de los sistemas-red, propiciando una nueva cobertura a sus necesidades (transformación de las valoraciones de los estímulos, y/o reorganizando el sistema al completo), o bien, transformando las estrategias, acciones y comportamientos de los sistemas en otras estrategias, acciones y comportamientos valorados como sinérgicos y recursivos de la atención de necesidades.

Si la regulación de un conflicto social conduce al sometimiento del uno sobre el otro, el modelo convivencial que se potenciará, podrá generar nuevos malestares en los sistemas, que impedirán o dificultarán la atención de las necesidades, articulando nuevos y mayores conflictos sociales. Si bien, esta realidad convivencial conflictiva violenta que se desarrolla en un espacio convivencial, no significa la supresión de todo espacio colaborativo, sinérgico y recursivo con el que dar atención a las necesidades, ni tampoco que sea esta realidad convivencial conflictiva eterna e inamovible. 
Es por ello que en este artículo se apuesta por un modelo de transformación del conflicto participativo-reflexivo-creativo encaminado a la potenciación de los espacios de atención sinérgica y recursiva de las necesidades, así como a la construcción de nuevas convivencias pacíficas, mediante el desarrollo de fórmulas convivenciales no violadoras, inhibidoras o pseudosatisfactoras, sino sinérgicas y recursivas con las que atender las necesidades de los múltiples y diversos sistemas humanos..

\section{A MODO DE CONCLUSIÓN}

De acuerdo con la premisa según la cual todo sistema humano vive en permanente desequilibrio sistémico (del caos al orden) en busca de estabilidad, cabe entenderse que tanto la construcción de paz como la regulación del conflicto social precisará que los sujetos sean capaces de elaborar estrategias e implementar acciones, aptas para fomentar la atención de las necesidades del conjunto de la población de manera sinérgica, lo que conllevará la configuración de morfologías convivenciales pacíficas en los diferentes espacios de cohabitación o interrelación humana.

Para ello, el modelo de Construcción Participada de Convivencias Pacíficas propone el análisis, para su transformación, tanto de las perturbaciones ocasionadas en los sistemas humanos que generan malestares o inquietudes que potencialmente podrían producir problemáticas y conflictos sociales; así como promover e impulsar procesos de relaciones convivenciales en los que sinérgicamente se favorezca la atención de las necesidades de unos y otros sistemas. Se favorezcan armónicos desequilibrios. Esto es, convivencias pacíficas.

Obvio es que la convivencia no está exenta de problemas socioculturales y conflictos producidos y re-producidos por los propios seres humanos, quienes, insertados en sus lógicas y esquemas de producción de conocimientos, son víctimas y verdugos de la producción y re-producción de las diferentes acciones vitales que dan nacimiento y continuación a los problemas y/o conflictos convivenciales. De hecho, las prácticas de los sujetos, orientadas a alcanzar el equilibrio interno de cada sistema, son también responsables de generar perturbaciones en otros sistemas, y, en ocasiones, la violación de la atención de las necesidades de éstos.

Ciertos modos de proceder de ciertos grupos sociales, o/y sujetos particulares, respecto de otros, producen considerables malestares en el camino de la búsqueda del armónico desequilibro: esclavos sobre esclavistas, señores feudales sobre siervos, propietarios del capital sobre trabajadores, nazis sobre judíos, etc.

Si se considera que las acciones de los seres humanos construyen internas realidades que se objetivan, y que requieren ser compatibilizadas en las redes 
en las que se está obligado a participar, huelga decir que la convivencia en comunidad, regida por el proceso de compatibilización de realidades, es, también, una construcción objetivada. Por tanto, es preciso aplicar un enfoque de investigación para la transformación de los conflictos y la construcción de paz, que se enmarque con la perspectiva sociopráxica; pues tanto las problemáticas socioculturales, como los conflictos sociales (y la paz), suceden en un marco relacional-convivencial, que los sujetos construyen y al que le otorgan un determinado valor.

La paz y el conflicto juegan un papel protagónico en el marco de la convivencia. La configuración de modelos convivenciales pacíficos y violentadores, son resultados que coexisten, se interrelacionan y retroalimentan. Es por ello que se considera pertinente el enfoque de paz imperfecta propuesto por Francisco Muñoz (2001), pues el adjetivo imperfecta, permite pensar en la emergencia simultánea de espacios convivenciales pacíficos y conflictos sociales. Pero además, desde el enfoque de paz transformadora, se asume la necesidad de generar procesos participativos de transformación de aquellas prácticas, valoraciones e impactos que propician la cristalización de modelos violentadores, en prácticas, valoraciones e impactos que propicien modelos sinérgicos de atención de las necesidades.

La cohabitación en los espacios de interrelación, implica que determinadas redes y sistemas sean a su vez constituyentes tanto de conflictos sociales como de espacios convivenciales pacíficos. Esta situación se origina dada la multidimensionalidad del sujeto, que en tanto universo en sí mismo, sus comportamientos, actitudes, acciones y valoraciones, responderán a sus propias partes constituyentes, tales como las redes y los contextos mediante los que construye y transforma su propia realidad.

Así, el sistema humano en permanente búsqueda de equilibrio interno, al recepcionar estímulos del medio en el que se desarrolla, procede a valorarlos, emitiendo respuestas con las que atender sus propias necesidades, conformando convivencias pacíficas o violentadoras, según sean dichos estímulos valorados por otros sistemas y redes. La emergencia en estos procesos vitales tanto de conflictos sociales, como de convivencias pacíficas, será una constante a regular por parte de los sistemas y sus redes. La concatenación de accionesrespuestas entre e intra redes y sistemas, producirá nuevos impactos en las relaciones y el potencial enredo de más sistemas y redes.

La toma en consideración de cada sistema y sus redes, las lógicas mediante las que se operan, los para qué y porqué de sus comportamientos y acciones, serán insumos sobre los que efectuar colectivas reflexiones intra e intereticulares, orientadas a consensuar nuevas estrategias, comportamientos y acciones 
capaces de atender las necesidades de los sistemas-redes de manera sinérgica y recursiva. Por ello, la mediación y la negociación-arbitraje se entienden como instrumentos válidos para el fomento de posibles acuerdos, recurriendo a segundas reflexividades que posibiliten la emergencia de nuevas energías colectivas, capaces de transformar conflictos, al tiempo que se potencia la creación de espacios de convivencias pacíficas.

Si se considera que son los sujetos los responsables de la producción de realidades, conocimientos, redes, y culturas, y que todo este conjunto de creaciones forman parte de la personal e intransferible cultura hologramática de cada cual, serán los sujetos los elementos clave en la producción de conflictos sociales y problemáticas socioculturales. Por tanto, es pertinente que sean éstos los principales protagonistas de cualquier proceso de transformación de los conflictos y producción de convivencias pacíficas en cualquier espacio de cohabitación existente. Formando parte de un proceso de reflexión colectiva respecto de los diferentes contextos que los condicionan, de uno u otro modo, a decantarse por unas u otras prácticas sociales. Prácticas sociales, que dirigidas a dar pertinente atención a las necesidades de cada cual, producen situaciones más o menos violadoras, inhibidoras, o, por el contrario, sinérgicas y recursivas con las que atender necesidades de los seres humanos con los que se convive

\section{BIBLIOGRAFÍA.}

Bertalanffy, L. y otros. (1978). Tendencias en la Teoría General de Sistemas. Madrid: Alianza. Diez, M. \& Mirón, M. (2004). "Una Paz Femenina”. En B. Molina y F. Muñoz (Coords.), Manual de Paz y Conflicto. Granada: Universidad de Granada, 67-94.

Dugan, M. (1996). "A Nested Theory of Conflict". A Leadership Jpurnal: Somen in Leadership-Sharing Vision", Volumen 1, 9-20.

Foerster, H. (1991). Las Semillas de la Cibernética. Barcelona: Gedisa.

Francés, F (2005). "Una aproximación al concepto de capital social a través de la técnica de modelos estructurales". En A. Alaminos, F. Francés, O. Santacreu (Eds.), Reflexiones teóricas y modelos empíricos sobre identidad, diversidad y participación social. I Jornadas Internacionales sobre el modelado estructural en el estudio de la realidad social. Alicante: OBETS Editorial, 93-117.

Galtung, J. (1985). Sobre la paz. Barcelona: Fontamara.

Galtung, J. (1995). Investigaciones Teóricas. Madrid: Tecnos.

Jiménez, F. (2009). "Hacia un Paradigma Pacífico: la Paz Neutra". Convergencia. Revista de Ciencias Sociales UAEM, Número Especial, 141-190.

Lederach, J. (1997). Construyendo la Paz, reconciliación sostenible en sociedades divididas. Bilbao: Gernika.

Maturana, H. Y Varela, F. (1990). El Árbol del Conocimiento. Madrid: Debate.

Matus, C. (2007). Teoría del Juego Social. Buenos Aires: Universidad Nacional de Lanus. 
Max-Neeff, Elizalde, y Hopenhayn (1986). Desarrollo a Escala Humana: una opción para el futuro. Barcelona: Icaria.

Médicos Del Mundo (2009). Violencia Contra la Mujer. Género, Cultura y Sociedades. [En línea] Available at: http://www.mdm-scd.org/files/GuidesVFF/GuideVFF_ES.pdf [Último acceso: Julio 2010].

Melillo, A. y Suarez Ojeda, E. (2001). Resilencia. Descubriendo las Propias Fortalezas. Buenos Aires: Paidós.

Montañés, M. (2006). Práxis Participativa Conversacional de la Producción de Conocimientos Sociocultural. Madrid: Tesis Doctoral, Universidad Complutense de Madrid.

Montañés, M. (2009). "La comunicación desde la perspectiva sociopráxica". Mediaciones Sociales. Revista de Ciencias Sociales y de la Comunicación, Número 4, 51-74.

Morin, E. (1995). Introducción al Pensamiento Complejo. Barcelona: Gedisa.

Muñoz, F. (2001). La Paz Imperfecta. Granada: Universidad de Granada.

Muñoz, F. (2004). "La paz". En B. Molina y F. Muñoz (coords.), Manual de Paz y Conflictos. Granada: Universidad de Granada, 21-42.

Paris y Martinez (2008). El papel de la sociedad civil en la transformación pacífica de conflictos. Barcelona: Icaria.

Prigogine, I. y Stengers, I. (1985). Order out of Chaos. Londres: Fontana.

Redorta, J. (2004). Cómo Analizar los Conflictos. La Tipología de Conflictos como Herramienta de Mediación. Barcelona: Paidós.

Szarazgat, D., Glaz, C. y Gaetano, C. (2009). "La Elocuencia de los Eufemismos. Un Análisis Semántico desde la Perspectiva Educativa". Revista Iberoamericana de Educación, Número 50, 221-232.

MANUEL MONTAÑÉS SERRANO es doctor y licenciado en Ciencias Políticas y Sociología por la UCM. Ha sido profesor en decenas de universidades, tanto españolas como latinoamericanas. En la actualidad es profesor en la Universidad de Valladolid (Campus de Segovia). Ha dirigido y realizado decenas de estudios. Siendo sus publicaciones más recientes, las siguientes: "Una estrategia participativa conversacional con la que producir conocimiento y propuestas de actuación sociocultural" en Revista de Antropología Experimental, n ${ }^{\circ}$ 12, 2012 , pp. 67-90. Redes acentradas (pág. 246-252) en Autogestión. UNILCO, Sevilla, 2012. "La incertidumbre Social" en Prisma Social" en Revista de Investigación Social, $\mathrm{n}^{\circ}$ 4, Junio, 2012. pp. 440-469. Metodología y Técnica participativa (Teoría y práctica de una estrategia de investigación participativa). UOC, Barcelona, 2009. "La comunicación desde la perspectiva sociopráxica" en Mediaciones Sociales. Revista de Ciencias Sociales y de la Comunicación, $n^{\circ} 4$, primer semestre de 2009, pp. 51-74. Ciudad, cultura, redes y participación en La ciudad a escala humana. UNILCO, Sevilla, 2007. "Más allá del debate cuantitativo/cualitativo: la necesidad de aplicar metodologías participativas conversacionales en Política y Sociedad, vol. 44. Núm 1, 2007. 
ESTEBAN A. RAMOS MUSLERA es licenciado en Ciencias Políticas (UCM) y Magíster en Investigación Participativa para el Desarrollo Local (UCM). En esto momento tiene depositada en la Universidad de Valladolid su Tesis Doctoral titulada Teoría y Práctica del Conflicto Social y la Paz desde la Perspectiva Sociopráxica. La Construcción Participada de Convivencias Pacíficas en Entornos Socialmente Vulnerables. En el campo profesional, ha sido técnico Superior en Investigación Social Participativa para EPSA Córdoba, en el marco del proyecto de desarrollo sociocomunitario en la barriada de Las Palmeras, Córdoba (desde 2007). En la actualidad es Coordinador de País de la ONGD Paz con Dignidad en Colombia. 